\title{
Cluster Properties of Lattice and Continuous Systems
}

\author{
M. Duneau and B. Souillard \\ Centre de Physique Théorique`, Ecole Polytechnique, F-91120 Palaiseau, France
}

\begin{abstract}
Various strong decay properties are proved for lattice systems with general $n$-body interactions, and for continuous systems with two-body and $n$-body interactions. The range of the potentials is finite or infinite.
\end{abstract}

\section{Introduction}

\section{Definitions $[1,2]$ :}

We say that the truncated correlation functions $\varrho_{A}^{T}$ satisfy a strong cluster property (S.C.P.) if there exists a real integrable function $U$ of the configuration space $\mathbb{R}^{v}$ or $\mathbb{Z}^{v}$ such that for any configuration $X$ (except perhaps a set of zero measure):

$$
\left|\varrho_{\Lambda}^{T}(X)\right| \leqq A \sum_{T \in \mathfrak{T}(X)} \prod_{\left(x, x^{\prime}\right) \in T} U\left(x, x^{\prime}\right)
$$

where the sum $\sum$ runs over all trees $T$ on $X$ (i.e. connected graphs without closed loop), and the product runs over all lines $\left(x, x^{\prime}\right)$ of the tree $T ; A$ and $U$ are independent of the box $\Lambda$, of $X$ and of the number of points $|X|$ of $X$, but depend on the potential $\Phi$ (including here the reciprocal temperature $\beta$ ) and on the activity $z$.

In the case of a lattice system, an equivalent formulation of S.C.P. can be given (for equivalence see Appendix).

$$
\left|\varrho_{\Lambda}^{T}(X)\right| \leqq A C^{|X|} \mathfrak{N}(X) e^{-L_{\delta}(X)}
$$

where $\mathfrak{N}(X)$ is a numerical factor equal to $N_{1} ! \ldots N_{p}$ ! when the points of $X$ occupy only $p$ different positions occuring respectively $N_{1}, \ldots, N_{p}$ times, $C$ is a constant and $L_{\delta}(X)$ is the shortest length with respect to some distance $\delta$ of all the trees constructed on the points of $X$ and arbitrary other points (for example $\delta\left(x, x^{\prime}\right)=$ $\chi\left|x-x^{\prime}\right|$ or $\left.\delta\left(x, x^{\prime}\right)=s \log \left(1+\alpha\left|x-x^{\prime}\right|\right), s>v\right)$, with $e^{-\delta\left(x, x^{\prime}\right)}$ integrable with respect to $x^{\prime} ; A, C$, and $\delta$ are again independent of $\Lambda, X$, and $|X|$ but depend on $\Phi$ and $z$.

Moreover the truncated correlations $\varrho_{A}^{T}$ are said to satisfy a strong decrease property (S.D.P.) if a bound of the type (1) holds, with a function $U\left(x-x^{\prime}\right)$ which is not integrable, or (2) with $s \leqq v$ or with a further multiplicative factor worse than $C^{|X|}$ (for instance $|X|$ !).

In a large number of situations with two-body potentials, S.C.P. have been proved $[2,3]$ to be equivalent to analyticity with respect to the activities (plus

* Equipe de Recherche du C.N.R.S. 174. 
some bound). Although they also take into account the separation of all points of $X$ with respect to each other, the S.D.P. have not such a property.

\section{Results of the Present Paper}

a) Lattice Systems with $n$-Body Interactions

For such general systems, only integrability is known [4]; for finite-range interactions a decrease like the diameter of $X$ has been proved [5]. In Part II, we prove S.C.P. for such systems with finite or infinite range interactions, if the potential decreases faster than $|x|^{-2 v}$ ( $v$ is the space dimension), and S.D.P. if the potential decreases slower than $|x|^{-2 v}$ but faster than $|x|^{-v}$. These results extend to $n$-body interactions the results of $[2,3]$ and we can also extend S.C.P. or S.D.P. in the whole analyticity region in the same situations as in these papers. Moreover, we can extend the equivalence between S.C.P. and analyticity (w.r. to the activities) to systems with finite-range or exponentially decreasing $n$-body potentials.

\section{b) Continuous Systems with 2-Body Interactions}

In that case, a decrease is known only for the 2-point function $\varrho^{T}\left(x_{1}, x_{2}\right)[6]$ and a S.C.P. is proved for the truncated correlations smeared with appropriate test functions $[2,3]$, yielding equivalence with analyticity w.r. to $z$. Here, in Part III, we prove S.C.P. for unsmeared correlations $\varrho_{A}^{T}$ at low activity, as soon as the potential decreases faster than $|x|^{-2 v}$. Moreover S.D.P. can be obtained in the domain of analyticity when some bound on the $\varrho_{A}^{T}(X)$ is known.

\section{c) Continuous Systems with $n$-Body Interactions}

For such systems, only existence of a unique state at low activity is known [7]. In Part IV, we prove S.D.P. of the $\varrho_{\Lambda}^{T}$ at low activity under some technical assumptions of regularity and decrease of the potential. This property extends again in the analyticity region. For truncated correlations smeared with appropriate test functions, S.C.P. can be derived at low activity and in the analyticity region, and they are again equivalent to analyticity for finite-range or exponentially decreasing interactions.

\section{Lattice Systems with $\boldsymbol{n}$-Body Interactions}

Let us consider a lattice system with an $n$-body interaction $\Phi$ defined for configurations $X,|X| \geqq 2$. Moreover we put $\Phi(x, x)=+\infty$ and let $\Phi\left(x, x, y_{1} \ldots y_{p}\right)$, $p \geqq 1$ be finite but arbitrary, and we suppose that:

$$
\operatorname{Sup}_{x \in \mathbb{Z}^{v}} \sum_{X: x \in X \subset \mathbb{Z}^{v}}|\Phi(X)|=D<\infty .
$$

It follows from $[4,5]$ that the correlation functions have the following series expansion for $\Lambda$ finite or infinite and $\xi=\operatorname{Sup}_{x \in \mathbb{Z}^{v}}\left|z_{x}\right|<\left[2 e^{D} e^{e^{D}-1}\right]^{-1}$ :

$$
\varrho_{\Lambda}(X ; \Phi, z)=z_{X} \sum_{n \geqq 0}(n !)^{-1} \sum_{Y \in \Lambda^{n}} z_{Y} \varphi(X ; Y)
$$


where $z_{X}\left[\right.$ resp. $\left.z_{Y}\right]$ means the product of the activities at the points of $X$, [resp. $\left.Y\right]$, and:

$$
\varphi(X ; Y)=\sum_{G \in \mathscr{G}_{c}(X ; Y)} \prod_{B \in G}\left(e^{-\Phi(B)}-1\right)
$$

where the sum runs over all generalized graphs $G=\left(B_{1}, \ldots, B_{k}\right), B_{i} \subset(X, Y),\left|B_{i}\right| \geqq 2$ and $B_{i} \neq B_{j}$ for $i \neq j$, connected with respect to the cluster $X$ and the points $Y$ (i.e. such that there exists a graph connected with respect to the cluster $X$ and the points of $Y$, for which each line links two points of some $B_{i}$ ).

The truncated correlations have then an analogous expansion in the same domain:

$$
\varrho_{\Lambda}^{T}(X ; \Phi, z)=z_{X} \sum_{n \geqq 0}(n !)^{-1} \sum_{Y \in \Lambda^{n}} z_{Y} \varphi(X, Y)
$$

with

$$
\varphi(X, Y)=\sum_{G \in \mathscr{G}_{c}(X, Y)} \prod_{B \in G}\left(e^{-\Phi(B)}-1\right)
$$

where the sum $\sum$ runs over all generalized graphs connected with respect to all the points of $X$ and $Y$.

The Ursell functions $\varphi(X ; Y)$ satisfy the following induction relation:

$$
\varphi(X ; Y)=e^{-W(X)} \sum_{Y^{\prime} \subset Y} K\left(X ; Y^{\prime}\right) \varphi\left(X_{1} Y^{\prime} ; Y \backslash Y^{\prime}\right)
$$

where $X_{1}$ is the set obtained by substracting from $X$ for example the first point, $x_{1}$, in the lexicographic order, and

$$
\begin{aligned}
& W(X)=\sum_{B: x_{1} \in B \subset X} \Phi(B) \\
& K(X ; Y)=\sum_{k \geqq 1} \sum_{\left\{Y_{1} \ldots Y_{k}\right\}^{Y}} \prod_{i=1}^{k}\left(e^{-W\left(X ; Y_{\imath}\right)}-1\right) .
\end{aligned}
$$

In this last expression, the sum $\sum$ runs over all coverings of $Y$ by $k$ non-empty, different (i.e. $Y_{i} \neq Y_{j}$ ) subsets, and

$$
W(X ; Y)=\sum_{B: x_{1} Y \subset B \subset X Y} \Phi(B) .
$$

Moreover the $K(X ; Y)$ satisfy the following integrability property [4]

$$
\sum_{Y \subset \mathbb{Z}^{\vee} \backslash X_{1}}|K(X ; Y)| \leqq 2 e^{e^{D}-1} .
$$

\section{Systems with Infinite Range Potentials}

Let us introduce for a given potential $\Phi$ all the distances $\delta$ on the configuration space, such that:

$$
|\Phi(B)|=e^{-L_{\delta}(B)} \Phi_{\delta}(B)
$$


(for instance $\delta\left(x, x^{\prime}\right)=\chi\left|x-x^{\prime}\right|$ or $\delta\left(x, x^{\prime}\right)=s \log \left(1+\alpha\left|x-x^{\prime}\right|\right)$ ), $L_{\delta}(B)$ is the minimal length with respect to the distance $\delta$ of the trees constructed on the points of $B$ and possibly arbitrary other vertices, and where:

$$
\operatorname{Sup}_{x \in \mathbb{Z}^{v}} \sum_{B: x \in B \subset \mathbb{Z}^{v}} \Phi_{\delta}(B)=D_{\delta}<\infty \text {. }
$$

Then the following theorem holds:

Theorem 1. If $\xi=\operatorname{Sup}\left|z_{x}\right|<C_{\delta}^{-1}$, with $C_{\delta}=2 e^{D_{\delta}} e^{e^{D_{\delta}}-1}$, then for $\Lambda$ finite or infinite:

$$
x \in \mathbb{Z}^{v}
$$

$$
\left|\varrho_{\Lambda}^{T}(X ; \Phi, z)\right| \leqq C_{\delta}^{-1}\left(\xi C_{\delta} /\left(1-\xi C_{\delta}\right)\right)^{|X|} \mathfrak{N}(X) e^{-L_{\delta}(X)} .
$$

(15) is a S.C.P. if $\delta\left(x, x^{\prime}\right)$ increases faster than $(v+\varepsilon) \log \left(1+\alpha\left|x-x^{\prime}\right|\right)$ with $\varepsilon>0$ and $\alpha>0$, and is a S.D.P. otherwise.

Remark. When the distance $\delta$ goes to zero $(\chi \rightarrow 0$ or $\alpha \rightarrow 0)$, then the radius $C_{\delta}^{-1}$ tends to the radius of convergence of the series expansion (4) and (6).

Proof. Introducing some obvious notations, the following inequalities follow from the definition (10), (11), and from (13): if $Y \subset \mathbb{Z}^{\nu} \backslash X$ :

$$
\begin{aligned}
|W(X ; Y)| \leqq & \sum_{\substack{B: \\
x_{1} Y \subset B \subset X Y}} e^{-L_{\delta}(B)} \Phi_{\delta}(B) \\
& \leqq e^{-L_{\delta}\left(x_{1}, Y\right)} W_{\delta}(X ; Y)
\end{aligned}
$$

Then, using $e^{a x}-1 \leqq x\left(e^{a}-1\right)$ for $x \in[0,1], a>0$ :

$$
e^{|W(X ; Y)|}-1 \leqq e^{-L_{\delta}\left(x_{1}, Y\right)}\left(e^{W \delta(X ; Y)}-1\right) .
$$

Hence

$$
\begin{aligned}
|K(X ; Y)| & \leqq \sum_{k \geqq 1} \sum_{\left\{Y_{1}, \ldots, Y_{k}\right\}^{Y}} \prod_{i=1}^{k} e^{-L_{\delta}\left(x_{1}, Y_{2}\right)}\left(e^{W_{\delta}\left(X ; Y_{\imath}\right)}-1\right) \\
& \leqq e^{-L_{\delta}\left(x_{1}, Y\right)} K_{\delta}(X ; Y)
\end{aligned}
$$

and since

$$
\begin{aligned}
& K(X ; Y)=-K\left(X ; x_{1} Y\right) \\
& |K(X ; Y)| \leqq e^{-L_{\delta}\left(x_{1}, Y\right)} K_{\delta}\left(X ; x_{1} Y\right)
\end{aligned}
$$

with

$$
K_{\delta}(X ; Y)=K_{\delta}\left(X ; x_{1} Y\right)=\sum_{k \geqq 1} \sum_{\left\{Y_{1}, \ldots, Y_{k}\right\}^{Y}} \prod_{i=1}^{k}\left(e^{W_{\delta}\left(X ; Y_{\iota}\right)}-1\right)
$$

for $Y \subset \mathbb{Z}^{v} \backslash X$, and

$$
K_{\delta}(X ; Y)=0 \text { for } Y \not \subset \mathbb{Z}^{\nu} \backslash X_{1} .
$$

Then

$$
\sum_{Y \subset \mathbb{Z}^{v} \backslash X_{1}} K_{\delta}(X ; Y) \leqq 2 e^{e^{D \delta}-1} .
$$


Now, we can define by induction the functions $\varphi_{\delta}(X ; Y)$ :

$$
\varphi_{\delta}(X ; Y)=e^{D} \sum_{Y^{\prime} \subset Y} K_{\delta}\left(X ; Y^{\prime}\right) \varphi_{\delta}\left(X_{1} Y^{\prime} ; Y \backslash Y^{\prime}\right)
$$

with $\varphi_{\delta}(\{x\})=1$;

Now, using the induction relations (8), (18), and the bounds (16) and (16') we obtain:

$$
|\varphi(X ; Y)| \leqq e^{-L_{\delta}(X ; Y)} \varphi_{\delta}(X ; Y)
$$

where $L_{\delta}(X ; Y)$ is the minimal length with respect to the distance $\delta$ of the trees constructed on the points of $X, Y$ and possibly arbitrary other vertices, which are connected w.r.t the cluster $X$ and the points of $Y$. Moreover, using (17), (18):

$$
\sum_{Y \in \mathbb{Z} v n} \varphi_{\delta}(X ; Y) \leqq C_{\delta}^{|X|+n-1} n !
$$

and consequently, expansion (6) gives

$$
\begin{aligned}
\left|\varrho_{\Lambda}^{T}(X ; \Phi, z)\right| & \leqq \xi^{|X|} e^{-L_{\delta}(X)} \sum_{n \geqq 0}\left(\xi^{n} / n !\right) \sum_{Y \in A^{n}} \varphi_{\delta}(X, Y) \\
& \leqq \xi^{|X|} e^{-L_{\delta}(X)} \sum_{n \geqq 0}\left(\xi^{n} \mathfrak{N}(X) / n !|X| !\right) \sum_{Y \in \Lambda^{|X|+n-1}} \varphi_{\delta}\left(x_{1}, Y\right) \\
& \leqq C_{\delta}^{-1}\left(\xi C_{\delta}\right)^{|X|} e^{-L_{\delta}(X)}(\mathfrak{N}(X) /|X| !) \sum_{n \geqq 0}((|X|+n-1) ! / n !)\left(\xi C_{\delta}\right)^{n}
\end{aligned}
$$

from which follows the theorem.

We indicate now a bound on the Ursell functions $\varphi(X ; Y)$ which generalises the one given in $[6,8]$ for two-body potentials, and which yields an alternative method to prove Theorem 1:

Theorem 2. The Ursell functions $\varphi(X ; Y)$ satisfy the following bound:

$$
|\varphi(X ; Y)| \leqq e^{(|X|+|Y|-1) D} \sum_{\tilde{T} \in \tilde{\mathfrak{z}}(X ; Y)} \prod_{(S, T) \in \tilde{T}}|K(S ; T)| .
$$

The sum $\Sigma$ runs over the generalized trees $\tilde{T}=\left\{\left(S_{1}, T_{1}\right), \ldots,\left(S_{k} T_{k}\right)\right\}$ of $\tilde{\mathfrak{I}}(X ; Y)$, which is defined by

$$
\tilde{\mathfrak{I}}(X ; Y)=\bigcup_{Y^{\prime} \subset Y} \bigcup_{\tilde{T} \in \tilde{\mathfrak{I}}\left(X_{1} Y^{\prime} ; Y \backslash Y^{\prime}\right)}\left\{\left(X, Y^{\prime}\right), \tilde{T}\right\}
$$

and $\mathfrak{I}(X ; \phi)=\phi, \tilde{\mathfrak{I}}(x ; y)=\{(x, y)\}$.

Proof. The bound (20) follows the induction relation (8) and from the definition (21).

\section{Systems with Finite Range Potentials}

In the case of finite range potentials (i.e. $\Phi(B)=0$ if $\sup _{x_{1}, x_{j} \in B}\left|x_{i}-x_{j}\right|>\lambda$ ), cluster properties follow easily from the fact that the Ursell functions $\varphi(X, Y)$ vanish if there are not enough points $Y$ to insure connexity between the points of $X$. With this method, weak decrease is obtained in [5] for lattice systems with $n$-body 
interactions. Of course S.C.P can be proved at the same time. We do not repeat here the proofs, see [2].

However we wish to indicate that one can get in some situations a stronger decrease by combining the method of Theorem 1 with the remark that

$$
\varphi(X, Y)=0 \quad \text { if } \quad|Y|<L(X) / \lambda-|X|+1
$$

where $L(X)$ is obtained with the euclidean distance. So let us consider the set of equalities for $\chi>0$ :

$$
|\Phi(B)|=e^{-\chi L(B)} \Phi_{\chi}(B)
$$

and let

$$
\begin{aligned}
& \operatorname{Sup}_{x \in \mathbb{Z}^{v}} \sum_{B: x \in B \subset \mathbb{Z}^{v}} \Phi_{\chi}(B)=D_{\chi} \\
& C_{\chi}=2 e^{D_{\chi}} e^{e^{D_{\chi}-1}} .
\end{aligned}
$$

Then we have the following theorem:

Theorem 3. Let $\xi=\operatorname{Sup}_{x \in \mathbb{Z}^{v}}\left|z_{x}\right|$. Then, for $\Lambda$ finite or infinite we have the following S.C.P. with all $\chi$ such that $\xi<C_{\chi}^{-1}$ :

$$
\left|\varrho_{\Lambda}^{T}(X ; \Phi, z)\right|<\left(\mathfrak{N}(X) / C_{\chi}\left(1-\alpha \xi C_{\chi}\right)(\alpha-1)^{|X|}\right) e^{-\left[\chi-\lambda^{-1} \log \alpha|z| C_{\chi}\right] L(X)}
$$

where $\alpha$ is any real number such that $1<\alpha<\left(\xi C_{\chi}\right)^{-1}$.

Remark. Depending on the potential, the better decrease may be obtained for $\chi=0$ (and the result is the same as using only (22)) or for some $\chi_{0}>0$.

\section{Decay Properties in Larger Domains}

For systems with finite-range or exponentially decreasing potentials, S.C.P can be proved also in the domain $\mathfrak{D}$ of analyticity with respect to $z$, if a bound of the type

$$
\left|\varrho_{\Lambda}^{T}(X)\right| \leqq A C^{|X|} \mathfrak{N}(X)
$$

holds in $\mathfrak{D}$ (for instance if the correlations are analytic in $\mathfrak{D}$ with respect to all $z_{x}, x \in \mathbb{Z}^{v}$, and if the one-point function $\varrho_{\Lambda}(x)$ is bounded in $\mathfrak{D}$; or if the partition function $Z_{\Lambda}\left(z_{x}, x \in \Lambda\right)$ does not vanish for $\Lambda$ large enough and all $z_{x}$ in $\left.\mathfrak{D}\right)$. The situations in which these hypothesis can be proved are the same as in $[2,3]$.

Then for systems with finite-range or exponentially decreasing potentials, we obtain as in $[2,3]$ the theorem of equivalence between S.C.P. and analyticity with respect to $z$. In these papers equivalence was proved between S.C.P. at real points $z$, and analyticity with respect to $z$ plus the bound (23). We only note here that as a matter of fact, equivalence holds between S.C.P. at real points, and analyticity with respect to the field of activities $z_{x}, x \in \mathbb{Z}^{v}$, plus a bound on the one-point function.

For systems with potentials decreasing like a power, only S.D.P. can be obtained outside the low activity region by the method of [3]. 


\section{Continuous Systems with 2-Body Potentials}

This part is concerned by a special proof of S.C.P. for continuous systems with 2-body stable potentials $\Phi\left(x_{1}, x_{2}\right)$ such that:

$$
\left|K\left(x_{1} x_{2}\right)\right|=\left|e^{-\beta \Phi\left(x_{1}, x_{2}\right)}-1\right| \leqq e^{-\delta\left(x_{1}, x_{2}\right)} K_{\delta}
$$

where $K_{\delta}$ is a constant and $\delta$ is a distance (assumed here for simplicity, to be translation invariant) such that:

$$
\int d x e^{-\frac{1}{2} \delta(0, x)}=C(\delta)<\infty .
$$

For instance, once again, $\delta(0, x)=\chi|x|$ or $\delta(0, x)=s \log (1+\alpha|x|), s>2 v, \alpha>0$.

Then we have the following

Theorem 4. The following S.C.P. holds for $|z|<r_{\delta}(\beta)=e^{-2 \beta B-1} \times K_{\delta}^{-1} C(\delta)^{-1}$, where $B$ is the constant of stability:

$$
\left|\varrho_{\Lambda}^{T}(X ; \beta, z)\right| \leqq A C^{|X|-1} \sum_{C \in \mathscr{C}(X)} \prod_{\left(x, x^{\prime}\right) \in C} U\left(x, x^{\prime} ; z\right)
$$

where $\mathscr{C}(X)$ is the set of all chains constructed on $X$, and

$$
A=2|z| e^{-2 \beta B}\left[1-|z| / r_{\delta}(\beta)\right]^{-2}, \quad C=|z| r_{\delta}(\beta) \cdot C_{\delta}^{-1}
$$

and

$$
U\left(x, x^{\prime} ; z\right)=\sum_{n \geqq 0} C^{n} \int_{\mathbb{R}^{n v}} d Y e^{-\frac{1}{2}\left[\delta\left(x, y_{1}\right)+\delta\left(y_{1}, y_{2}\right)+\ldots+\delta\left(y_{n}, x^{\prime}\right)\right]} .
$$

If the potential $\Phi$ decreases exponentially, $U\left(x, x^{\prime} ; z\right)$ is an exponentially decreasing function of $\left|x-x^{\prime}\right|$.

If the potential $\Phi$ decreases like a power $|x|^{-s}, U\left(x, x^{\prime} ; z\right)$ decreases at least like $|x|^{-s / 2}$.

To prove this result, we first prove the following lemma:

Lemma 5. Let $\mathfrak{T}(X),($ resp. $\mathscr{C}(X))$ be the set of all trees (resp. chains) constructed on the points of $X$, and let $L_{\delta}(T)$ (resp. $\left.L_{\delta}(C)\right)$ be the length with respect to the distance $\delta$ of the tree $T$ (resp. the chain $C$ ).

Then:

$$
\sum_{T \in \mathfrak{I}(X)} e^{-L_{\delta}(T)} \leqq 2\left(|X|^{|X|-2} /|X| !\right) \sum_{C \in \mathscr{C}(X)} e^{-\frac{1}{2} L_{\hat{\delta}}(C)} .
$$

Proof of Lemma 5. Let us consider the set $\mathscr{C}_{T}$ of all chains which can be associated to the three $T \in \mathfrak{I}(X)$ by "turning around" $T$ and keeping each point $x_{i} \in X$ once and only once as soon as we meet it: (see for instance Fig. 1).

Fig. 1

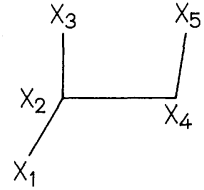

Tree

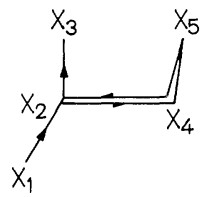

$x_{1}$

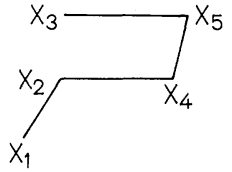

Chain 
Then $\forall C \in \mathscr{C}_{T}, 2 L_{\delta}(T) \leqq L_{\delta}(C)$.

Let now $v(T)$ be the number of distinct chains associated with the tree $T$; then:

$$
\sum_{T \in \mathfrak{T}(X)} e^{-L_{\delta}(T)} \leqq \sum_{C \in \mathscr{G}(X)} e^{-\frac{1}{2} L_{\delta}(C)} \sum_{T: C \in \mathscr{C}_{T}} v(T)^{-1} .
$$

The last factor will be computed using this equation with $\delta \equiv 0$, and using the symmetry with respect to permutations of points:

$$
\sum_{T \in \mathfrak{I}(X)} 1=|X|^{|X|-2}=\sum_{C \in \mathscr{C}(X)} \sum_{T: C \in \mathscr{C}_{T}} v(T)^{-1}
$$

and then

$$
\sum_{T: C \in \mathscr{C}_{T}} v(T)^{-1}=2|X|^{|X|-2} /|X| !
$$

which ends the proof of Lemma 5.

Proof of Theorem 4. The Ursell functions satisfy the following bound, proved in $[6,8]$ for positive and hard-core potentials and in [3] for general stable potentials:

$$
|\varphi(X)| \leqq e^{2 \beta B|X|-2} \sum_{T \in \mathfrak{T}(X)} \prod_{\left(x, x^{\prime}\right) \in T}\left|K\left(x, x^{\prime}\right)\right| .
$$

In view of Lemma 5 and using (24), we obtain:

$$
\begin{aligned}
|\varphi(X, Y)| \leqq & 2\left(e^{2 \beta B}\right)^{|X|+|Y|-2} K_{\delta}^{|X|+|Y|-1} \\
& \cdot\left((|X|+|Y|)^{|X|+|Y|-2} /(|X|+|Y|) !\right) \sum_{C \in \mathscr{C}(X, Y)} e^{-\frac{1}{2} L_{\delta}(C)} .
\end{aligned}
$$

We can always associate a chain on $X$ to each chain on $X, Y$ and then we can obtain a bound on the coefficients of the series expansion of $\varrho^{T}(X)$ :

$$
n !^{-1} \int_{\mathbb{R}^{n \nu}} d Y \sum_{C \in \mathscr{C}(X, Y)} e^{-\frac{1}{2} L_{\delta}(C)}=\sum_{C \in \mathscr{C}(X)} \sum_{\substack{\left\{n_{0}, \ldots, n_{1}, \ldots \mid\right\}: \\ n_{l} \geqq 0, \sum n_{l}=n}} \prod_{i=0}^{|X|} \int_{\mathbb{R}^{n} n_{i}^{\nu}} d Y e^{-\frac{1}{2} L_{\delta}\left(C_{i}\right)}
$$

where $C_{i}(1 \leqq i<|X|)$ is the chain joining the $i^{\text {th }}$ point of $C$ to its $(i+1)^{\text {th }}$ point with $n_{i}$ intermediate points $y ; C_{0}\left[\operatorname{resp} C_{|X|}\right]$ is a chain constructed on the first [resp. the last] point of $C$ with $n_{0}$ [resp. $\left.n_{|X|}\right]$ points $y$.

The bound (26) then follows from (29), (30), from the series expression of the $\varrho^{T}(X)$, and from the inequality:

$$
(N+n)^{N+n-2} \leqq(N+n-1)^{N+n-1}<e^{N+n-1}(N+n) !
$$

for $N \geqq 2$ and $n \geqq 0$.

The announced behaviour of $U\left(x, x^{\prime} ; z\right)$ can be obtained for example by considering its Fourier transform in the case of an exponentially decaying potential, and may be checked from direct computation for potentials decaying like a power. 


\section{Extensions in the Domain of Analyticity}

We mention here that using methods of [3], S.D.P. can be obtained in the region of analyticity with respect to $z$ if moreover some bound of the type

$$
\left|\varrho_{\Lambda}^{T}(X ; \beta, z)\right| \leqq A(|X|)
$$

is known. In such cases however (i.e. continuous systems), only S.D.P. and not S.C.P. can be obtained, up to now, outside the low activity region.

On the other hand we recall $[2,3]$ that the truncated correlations smeared with some test functions satisfy S.C.P. outside the low activity region and such property is then equivalent to analyticity with respect to $z$ plus bounds.

\section{Continuous Systems with $\boldsymbol{n}$-Body Interactions}

Under conditions of ultra-stability and regularity of the potential, the existence of a unique thermodynamic state has been proved [7] at low activity for continuous systems with $n$-body interactions. In this section we shall give results on the decay of correlations under reasonable conditions on the potential.

Let us introduce:

$$
\begin{aligned}
\varphi_{\mu}(X ; Y) & =e^{\mu W(X)} \varphi(X, Y) \\
K_{\mu}(X ; Y) & =e^{-(1-\mu) W(X)-\mu W\left(X_{1}, Y\right)} K(X ; Y)
\end{aligned}
$$

and remark that

$$
\varphi(X, Y)=\varphi_{\mu}(X, Y) \text {. }
$$

The potential is said to be regular [7], if for some $\mu, 0<\mu<1$ and $\beta$ sufficiently small:

a) $\left|K_{\mu}(X ; Y)\right|$ is integrable with respect to $Y$ in every $\Lambda$, except for $X$ in a set of Lebesgue-measure zero.

b) Except for $X$ in a set of Lebesgue-measure zero, if $W(X)=\infty$, then $W(X, Y)>-\infty$ almost everywhere (with respect to $Y$ )

c)

$$
\lim _{\Lambda \rightarrow \infty} \operatorname{ess} \sup \sum_{X \subset \Lambda}^{\infty} \int d Y_{n} n !^{-1}\left|K_{\mu}(X ; Y)\right|=D_{\mu}<\infty .
$$

\section{Series Expansion and Ursell Functions}

By same methods as usual, one can show that the truncated correlations are again given by the series expansion:

$$
\varrho_{\Lambda}^{T}(X ; \Phi, z)=z^{|X|} \sum_{n \geqq 0} z^{n} n !^{-1} \int_{\Lambda^{n}} \varphi(X, Y) d Y
$$

and that the Ursell functions $\varphi_{\mu}(X ; Y)$ satisfy the following induction relation:

$$
\varphi_{\mu}(X ; Y)=\sum_{Y^{\prime} \subset Y} K_{\mu}\left(X ; Y^{\prime}\right) \varphi_{\mu}\left(X_{1} Y^{\prime} ; Y \backslash Y^{\prime}\right)
$$


Then we have

Theorem 6. If $\Phi$ is a regular potential, the following S.C.P. holds

$$
\left|\varphi_{\mu}(X ; Y)\right| \leqq \sum_{\tilde{T} \in \tilde{\mathfrak{z}}(X ; Y)} \prod_{(S . T) \in \tilde{T}}\left|K_{\mu}(S ; T)\right|
$$

where the sum $\Sigma$ runs over the generalized trees $\tilde{T}$ defined in Theorem 2.

Moreover using also (33), one easily checks that:

$$
\int\left|\varphi_{\mu}\left(X ; Y_{n}\right)\right| d Y_{n} \leqq D_{\mu}^{|X|+n-1} n !
$$

which insures the absolute convergence of (32) for $|z|<D_{\mu}^{-1}$.

\section{Systems with Finite-Range Potentials}

If the potential has a finite-range $\lambda$, and is regular, one can prove a S.C.P. on the smeared truncated correlations, and using the strong regularity condition (see below), a S.D.P of the unsmeared truncated correlations.

Let us say that a potential $\Phi$ is strongly regular if, besides its regularity, it satisfies:

$$
\lim _{\Lambda \rightarrow \infty} \operatorname{ess}_{\substack{X_{0} \subset A \\ X \subset \Lambda}} \sum_{n=0}^{\infty} \int d Y_{n} n !^{-1}\left|K_{\mu}\left(X_{0} ; X, Y_{n}\right)\right| \leqq K_{\mu}<\infty .
$$

Let us moreover define the smeared truncated correlation by using for example a discretisation of $\mathbb{R}^{v}$ by hypercubes of side a and centered at $a \cdot \bar{x}, \bar{x} \in \mathbb{Z}^{v}$ :

$$
\bar{\varrho}_{\Lambda}^{T}(a \bar{X} ; \Phi, z)=\int_{\mathbb{R}^{v \mid} \mid X_{1}} d X^{\prime} \varrho_{\Lambda}^{T}\left(X^{\prime} ; \Phi, z\right) \prod_{\bar{x}_{l} \in \bar{X}} \chi_{a \bar{x}_{t}}\left(x_{i}^{\prime}\right) / a^{v}
$$

where $\chi_{a \bar{x}}$ is the characteristic function of the hypercube centered at $a \cdot \bar{x}$.

Then, the following theorem holds:

Theorem 7. If the potential $\Phi$ is regular and if $|z|<D_{\mu}^{-1}$, then the S.C.P holds, for any $\alpha$ such that $1<\alpha<\left(|z| D_{\mu}\right)^{-1}$ :

$$
\left|\varrho_{\Lambda}^{T}(a \bar{X} ; \Phi, z)\right| \leqq \mathfrak{N}(\bar{X}) D_{\mu}^{-1}\left(1-\alpha|z| D_{\mu}\right)^{-1}(\alpha-1)^{-|\bar{X}|}\left(\alpha|z| D_{\mu}\right)^{\frac{\bar{L}(a \bar{X})}{\lambda}}
$$

where $\bar{L}(a \bar{X})=\operatorname{Inf}\left\{L\left(X^{\prime}\right): X^{\prime} \in \mathbb{R}^{v|\bar{X}|}\right.$ and $\left.\prod_{i=1}^{|\bar{X}|} \chi_{a \bar{x}_{l}}\left(\chi_{i}^{\prime}\right)=1\right\}$.

If the potential $\Phi$ is strongly regular and if $|z|<\left(e K_{\mu}\right)^{-1}$, a S.D.P. holds:

$$
\left|\varrho_{\Lambda}^{T}(X ; \Phi, z)\right| \leqq|X| !\left(|z| e K_{\mu}\right)^{\frac{L(X)}{\lambda}} /\left(1-|z| e K_{\mu}\right) .
$$

Proof of Theorem 7. The proof is derived from the induction relation (33) using (35) for the first part and for the second part:

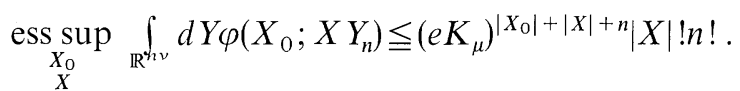




\section{Systems with Infinite-Range Potentials, and Extensions}

Assume, as an extension of the discret case, that there exist distances $\delta$ on $\mathbb{R}^{v}$ such that

$$
\left|K_{\mu}(X ; Y)\right|=e^{-L_{\hat{\delta}}\left(x_{1}, Y\right)} K_{\mu, \delta}(X ; Y),
$$

where $K_{\mu, \delta}$ satisfies $c$ ) of the regularity condition.

Then using the same methods as in the previous parts, one obtains S.C.P at small activity for smeared correlations $\varrho_{\Lambda}^{T}(a \bar{X} ; \Phi, z)$.

If furthermore $K_{\mu, \delta}$ satisfies a condition of the type (36), one obtains S.D.P on (unsmeared) truncated correlations $\varrho_{\Lambda}^{T}(X ; \Phi, z)$.

Moreover, as previously, one can derive S.C.P or S.D.P in the domain of analyticity with respect to $z$.

\section{Appendix}

In the appendix, we want to prove that the bound (2) given for the lattice systems is a S.C.P as soon as $e^{-\delta\left(x, x^{\prime}\right)}$ is integrable. As a matter of fact the inequality $\frac{1}{2} \hat{L}_{\delta}(X) \leqq L_{\delta}(X) \leqq \hat{L}_{\delta}(X)$, where $\hat{L}_{\delta}(X)$ is the length of the minimal tree constructed on points $X$ (without additional vertices), insures $[1,2]$ the result if the distance $\delta$ is euclidean:

$$
\delta\left(x, x^{\prime}\right)=\chi\left|x-x^{\prime}\right| \quad \text { or if } \delta\left(x, x^{\prime}\right)=s \log \left(1+\alpha\left|x-x^{\prime}\right|\right),
$$

with $s>2 v$. But the bound (2) is not obviously integrable for

$$
\delta\left(x, x^{\prime}\right)=s \log \left(1+\alpha\left|x-x^{\prime}\right|\right), \quad v<s \leqq 2 v .
$$

We solve here this problem by proving the following theorem for any logarithmic distance:

Theorem ${ }^{1}$. Let $\delta\left(x, x^{\prime}\right)=s \log \left(1+\alpha\left|x-x^{\prime}\right|\right)$ and $\delta^{\prime}\left(x, x^{\prime}\right)=s \log \left(1+\alpha\left|x-x^{\prime}\right| / 2\right)$.

Then $L_{\delta}(X) \geqq \hat{L}_{\delta^{\prime}}(X)$.

Proof. We shall show that for any tree $T$ constructed on points $X$ and additional points $Y$, one can construct a tree $\hat{T}$ on $X$, whose length with respect to $\delta^{\prime}$ is less that the length of $T$ with respect to $\delta$.

It is clearly sufficient to prove that:

$$
\prod_{l \in T}(1+\alpha|l|) \geqq \prod_{i \in \hat{T}}(1+\alpha|\hat{l}| / 2) \text {. }
$$

Let us consider a star consisting of a point $y$ linked to $N$ points $x_{1} \ldots x_{N}$ ordered in a way such that:

$$
\left|y-x_{1}\right| \geqq\left|y-x_{2}\right| \geqq \ldots \geqq\left|y-x_{N}\right|
$$

then

$$
\begin{aligned}
& \left|y-x_{1}\right| \geqq \frac{1}{2}\left|x_{1}-x_{2}\right| \\
& \left|y-x_{2}\right| \geqq \frac{1}{2}\left|x_{2}-x_{3}\right| \\
& ------------ \\
& \left|y-x_{N-1}\right| \geqq \frac{1}{2}\left|x_{N-1}-x_{N}\right| .
\end{aligned}
$$

\footnotetext{
This result was obtained with C. de Calan.
} 


\section{So that}

$$
\prod_{i=1}^{N}\left(1+\alpha\left|y-x_{i}\right|\right) \geqq \prod_{i=1}^{N-1}\left(1+\alpha\left|x_{i}-x_{i+1}\right| / 2\right) \cdot\left(1+\alpha\left|y-x_{N}\right|\right) .
$$

Now consider the tree $T$ on $X$ and additional points $Y$. We first suppress all points of $Y$ which are extremities, and the corresponding factors larger than 1 . Now on the new tree thus obtained, we consider any point $y$ which is linked at most to one other point $y^{\prime}$ of $Y$ and certainly to one or more points $x_{1} \ldots x_{n}$ of $X$. Then using the previous remark we minimize the contribution of the star $(y, x),, \ldots,\left(y, x_{n}\right)$ by the contribution of a chain with factors $1+\alpha\left|x_{i}-x_{i+1}\right| / 2$; the remaining line $\left(y, x_{n}\right)$ is to be associated with a further line $\left(y, y^{\prime}\right)$ or $(y, x)$, and is replaced by a line $\left(x_{n}, y^{\prime}\right)$ or $\left(x_{n}, x\right)$, using:

$$
\begin{aligned}
& \left(1+\alpha\left|y-x_{n}\right|\right)\left(1+\alpha\left|y-y^{\prime}\right|\right) \geqq 1+\alpha\left|x_{n}-y^{\prime}\right| \\
& \text { or } \\
& \left(1+\alpha\left|y-x_{n}\right|\right)(1+\alpha|y-x|) \geqq 1+\alpha\left|x_{n}-x\right| \geqq 1+\alpha\left|x_{n}-x\right| / 2 .
\end{aligned}
$$

So that we have suppressed one point $y$ and by induction, we can construct a tree $\hat{T}$ on $X$. Notice that the induction is possible since $T$ being a tree there exists at each step at least one point $y$ linked to at most one other point $y^{\prime}$. This induction procedure ends the proof.

Acknowledgements. We are indebted to professors C. de Calan, G. Gallavotti and D. Iagolnitzer for interesting discussions.

\section{References}

1. Duneau, M., Iagolnitzer, D., Souillard, B.: Commun. math. Phys. 31, 191 (1973)

2. Duneau, M., Iagolnitzer, D., Souillard, B.: Commun. math. Phys. 35, 307 (1974)

3. Duneau, M., Souillard, B., Iagolnitzer, D.: J. Math. Phys. 16, 1662 (1975)

4. Gallavotti, G., Miracle-Sole, S.: Commun. math. Phys. 7, 274 (1968)

5. Del Grosso, G.: Commun. math. Phys. 37, 141 (1974)

6. Groeneveld, J.: Lecture Notes of conference on Graph Theory and Theoretical physics, Frascati, Italy, 1964 and in: Statistical Mechanics: foundations and applications (ed. A. Bak). New York: Benjamin 1967

7. Greenberg, W.: Commun. math. Phys. 22, 259 (1971)

8. Penrose, O.: In: Statistical Mechanics, foundations and applications (ed. A.Bak). New York: Benjamin 1967

Communicated by G. Gallavotti

Received August 3, 1975 